\title{
Weil restriction and the Quot scheme
}

\author{
Roy Mikael Skjelnes
}

\begin{abstract}
We introduce a concept that we call module restriction, which generalizes the classical Weil restriction. After having established some fundamental properties as existence and étaleness, we apply our results to show that the Quot functor Quot $_{F_{X} / S}^{n}$ of Grothendieck is representable by an algebraic space for any quasi-coherent sheaf $F_{X}$ on any separated algebraic space $X \longrightarrow S$.
\end{abstract}

\section{Introduction}

The main novelty in this article is the introduction of the module restriction, which is a generalization of the classical Weil restriction. Our main motivation for introducing the module restriction is given by our application to the Quot functor of Grothendieck.

If $F_{X}$ is a quasi-coherent sheaf on a scheme $X \longrightarrow S$, then the Quot functor Quot $_{F_{X} / S}$ parametrizes quasi-coherent quotients of $F_{X}$ that are flat and have proper support over the base. When the scheme $X \longrightarrow S$ is projective, the base is Noetherian and $F_{X}$ is coherent, the Quot functor is represented by a scheme given as a disjoint union of projective schemes [Gro95]. When $X \longrightarrow S$ is locally of finite type and separated, Artin showed that the Quot functor is representable by an algebraic space (see [Art69] and the erratum in [Art74]). A generalization to quasi-coherent $F_{X}$ on projective schemes was carried out in [DB14], and algebraicity of the Quot functor in general was proven quite recently in [HR15].

When the fixed sheaf $F_{X}$ is the structure sheaf $\mathscr{O}_{X}$ of $X$, the Quot functor is referred to as the Hilbert functor $\underline{H i l b}_{X / S}$.

Grothendieck, who both introduced the Quot functor and showed representability in the projective situation, also pointed out the connection between the Hilbert scheme and the Weil restriction [Gro95, Section 4]. If $f: Y \longrightarrow X$ is a morphism with $X$ separated, there is an open subset $\Omega_{Y \rightarrow X}$ of $\underline{\operatorname{Hilb}}_{Y / S}$ from where the push-forward map $f_{*}$ is defined. The fibers of $f_{*}: \Omega_{Y \rightarrow X} \longrightarrow \underline{\text { Hilb }}_{X / S}$ are identified with the Weil restrictions.

However, even though the Weil restriction appears naturally in connection with Hilbert schemes, there does not seem to exist any description of the more general situation with the Quot scheme replacing the Hilbert scheme. The purpose of this article is to give such a description with the Quot functor in the zero-dimensional case. We say that a quasi-coherent and finite type sheaf $\mathscr{E}$ on $g: X \longrightarrow S$ is finite, flat of relative rank $n$ if the $\operatorname{support} \operatorname{Supp}(\mathscr{E})$ is affine over $S$

Received 11 March 2013, accepted in final form 8 April 2015.

2010 Mathematics Subject Classification 14A20, 14C05, 14D22.

Keywords: Weil restriction, Quot scheme, Fitting ideals.

This journal is (C) Foundation Compositio Mathematica 2015. This article is distributed with Open Access under the terms of the Creative Commons Attribution Non-Commercial License, which permits non-commercial reuse, distribution, and reproduction in any medium, provided that the original work is properly cited. For commercial re-use, please contact the Foundation Compositio Mathematica. 


\section{WeIL RESTRICTION AND THE QUOT SCHEME}

and $g_{*} \mathscr{E}$ is locally free of rank $n$. We denote by $\underline{\text { Quot }}_{F_{X} / S}^{n}$ the functor parametrizing quotients of $F_{X}$ that are finite, flat and of rank $n$.

The Weil restrictions were generalized in [Ols06] and [AGV73], but those generalizations are not suited for the present discussion. The generalization we undertake here is in the direction from ideals to modules, and is as follows. Fix a homomorphism of $A$-algebras $f: B \longrightarrow R$ and a $B$-module $M$. The module restriction $\mathscr{M} \mathrm{od}_{B \rightarrow R}^{M}$ parametrizes, as a functor from $A$-algebras to sets, the $R$-module structures extending the fixed $B$-module structure on $M$.

More specifically, let $\mu: B \longrightarrow \operatorname{End}_{A}(M)$ denote the $A$-algebra homomorphism corresponding to the $B$-module structure on $M$. Then the module restriction $\mathscr{M} \operatorname{od}_{B \rightarrow R}^{M}$ parametrizes $A$-algebra homomorphisms $\xi: R \longrightarrow \operatorname{End}_{A}(M)$ such that $\xi \circ f=\mu$, where $f: B \longrightarrow R$ is the fixed structure map.

The appearance of the non-commutative $\operatorname{ring} \operatorname{End}_{A}(M)$ is natural in this context, and is also the cause of some of the technical complications that have to be addressed.

When $M$ is finitely generated and projective as an $A$-module, we show that the module restriction $\mathscr{M} \operatorname{od}_{B \rightarrow R}^{M}$ is representable by an $A$-algebra. We show representability by constructing the representing object in the free algebra situation, and using Fitting ideals in the general situation.

These observations are summarized by the following result.

Theorem 1.1. Let $X \longrightarrow S$ be a separated morphism of schemes (or algebraic spaces), and let $\mathscr{C} \mathrm{oh}_{X / S}^{n}$ denote the stack of quasi-coherent sheaves on $X$ that are finite, flat of relative rank $n$ over the base $S$. For any affine morphism $f: Y \longrightarrow X$ the push-forward map

$$
f_{*}: \mathscr{C} \mathrm{oh}_{Y / S}^{n} \longrightarrow \mathscr{C} \mathrm{oh}_{X / S}^{n}
$$

is schematically representable.

The fibers of the push-forward map (1.1) are the module restrictions parametrizing sheaves $\mathscr{F}$ on $Y$ that are finite, flat and of relative rank $n$ over the base, and such that the push-forward $f_{*} \mathscr{F}$ is isomorphic to a fixed sheaf $\mathscr{E}$ on $X$.

When the morphism $f: Y \longrightarrow X$ is étale, the push-forward map (1.1) is not in general étale. The map (1.1) is étale when restricted to the open substack $\mathscr{U}_{Y \rightarrow X}$ consisting of sheaves $\mathscr{F} \in \mathscr{C}$ oh ${ }_{Y / S}^{n}$ on $Y$ such that the induced map of supports

$$
\operatorname{Supp}(\mathscr{F}) \longrightarrow \operatorname{Supp}\left(f_{*} \mathscr{F}\right)
$$

is an isomorphism.

The requirement concerning the support of the sheaves highlights a difference between the Quot functor and the Hilbert functor. Fibers of (1.1) over structure sheaves $\mathscr{O}_{Z}$ of closed subschemes $Z \subseteq X$ are étale when $f: Y \longrightarrow X$ is étale.

Let $f_{*}: \mathscr{U}_{Y \rightarrow X} \longrightarrow \mathscr{C}_{\mathrm{oh}_{X / S}^{n}}$ also denote the restriction of (1.1) to the open substack where the induced map of supports is an isomorphism. If we denote $Z=\operatorname{Supp}(\mathscr{E})$ the support of a given element $\mathscr{E}$ in $\mathscr{C} \mathrm{oh}_{X / S}^{n}$, then we show that the fiber $f_{*}^{-1}(\mathscr{E})$ equals the Weil restriction of $Y \times_{X} Z \longrightarrow Z$. Thus, the Weil restriction appears naturally in the more general context with the Quot functors as well. Even though the support $Z=\operatorname{Supp}(\mathscr{E}) \longrightarrow S$ is not necessarily flat, it turns out that in our situation the Weil restriction of $Y \times_{X} Z \longrightarrow Z$ still exists as a scheme.

Having established these technical results concerning the support, the representability of Quot $_{F_{X} / S}^{n}$ follows easily. Let $F_{Y}$ denote the pull-back of the quasi-coherent sheaf $F_{X}$ along 


\section{R. M. SkJELnes}

$f: Y \longrightarrow X$. There is a natural forgetful map $\underline{\mathrm{Quot}}_{F_{X} / S}^{n} \longrightarrow \mathscr{C} \mathrm{oh}_{X / S}^{n}$ whose pull-back along the map (1.1) restricted to $\mathscr{U}_{Y \rightarrow X}$ gives a representable étale covering

$$
\Omega_{Y \rightarrow X}^{F} \longrightarrow \underline{\text { Quot }}_{F X}^{n} / S \text {. }
$$

This étale covering specializes in the Hilbert functor situation, that is, with $F_{X}=\mathscr{O}_{X}$, to the classical covering mentioned earlier, with the fibers being Weil restriction. We obtain the following result.

Theorem 1.2. Let $X \longrightarrow S$ be a separated map of algebraic spaces, and let $F_{X}$ be a quasicoherent sheaf on $X$. Then the Quot functor $\underline{\text { Quot }}_{F_{X} / S}^{n}$ is representable by an algebraic space.

In particular, this generalizes the result on the representability of the Hilbert functor $\underline{H i l b}_{X / S}^{n}$ described in [ES14]. See also the generalization to Hilbert stacks in [Ryd11]. The result also extends the earlier mentioned result of Artin in the sense that we do not assume the map $X \longrightarrow S$ to be of finite type and that there is no restriction on the base $S$.

\section{Fitting ideals}

We will in this first section point out some facts about Fitting ideals that we will use later on.

2.1. Conventions. A commutative $\operatorname{ring} A$ is always a unital commutative ring. By the category of $A$-algebras, we mean the category of commutative $A$-algebras.

Lemma 2.2. Let $E$ be a projective $A$-module of rank $n$. Let $E \longrightarrow Q$ be a quotient module, and let $F_{n-1}(Q) \subseteq A$ denote the $(n-1)$ st Fitting ideal of $Q$. Then the $A$-module map $E \longrightarrow Q$ is an isomorphism if and only if $F_{n-1}(Q)=0$. In particular, a ring homomorphism $A \longrightarrow A^{\prime}$ will factor through $A / F_{n-1}(Q)$ if and only if $E \otimes_{A} A^{\prime} \longrightarrow Q \otimes_{A} A^{\prime}$ is an isomorphism.

Proof. The statement can be checked locally on $A$; hence we may assume that $E$ is free of finite rank $n$. The result then follows from the definition of the Fitting ideal.

2.3. Rank of projective modules. The rank of a finitely generated projective module $E$ is constant on the connected components of $\operatorname{Spec}(A)$. We will employ the following notation. Let $I \subseteq E$ be a submodule of a finitely generated and projective module $E$. We let

$$
\operatorname{Fitt}(I):=F_{\mathrm{rk} E-1}(E / I) \subseteq A
$$

denote the Fitting ideal we obtain by assigning on each connected component of $\operatorname{Spec}(A)$ the Fitting ideal $F_{n-1}(E / I)$, where $n$ is the rank of $E$ on that particular component.

2.4. Trace map. Let $E$ be an $A$-module. The dual module $\operatorname{Hom}_{A}(E, A)$, we will denote by $E^{\star}$. The trace map is the induced $A$-module homomorphism

$$
\operatorname{Tr}: E \otimes_{A} E^{\star} \longrightarrow A \text {. }
$$

LEMma 2.5. Let $I \subseteq E$ be an inclusion of $A$-modules, where $E$ is finitely generated and projective. Then we have the identity of ideals $\operatorname{Tr}\left(I \otimes_{A} E^{\star}\right)=\operatorname{Fitt}(I)$ in $A$, where $\operatorname{Tr}$ denotes the trace $\operatorname{map}(2.1)$.

Proof. Both the Fitting ideal and the trace map Tr commute with base change, and we may therefore assume that $E$ is free as an $A$-module. The statement is then clear. 


\section{WeIL RESTRICTION AND THE QUOT SCHEME}

2.6. Closed conditions. Let $F$ be a co-variant functor from the category of $A$-algebras (or a subcategory of it) to the category Sets of sets. We say that $F$ is a closed condition on $A$ if the functor $F$ is representable by a quotient algebra of $A$.

Proposition 2.7. Let $\xi: R \longrightarrow E$ be an $A$-module homomorphism. Assume that $E$ is finitely generated and projective as an $A$-module.

(i) Let $I \subseteq R$ be a submodule. Then $\xi$ factoring through the quotient map $R \longrightarrow R / I$ is a closed condition on $A$.

(ii) Let $\xi^{\prime}: R \longrightarrow E$ be another $A$-module homomorphism. Then $\xi$ being equal to $\xi^{\prime}$ is a closed condition on $A$.

Proof. In the first situation consider the Fitting ideal Fitt $\left(I_{1}\right)$ of the quotient module of $E$ given by $I_{1}=\xi(I)$. In the second situation consider the Fitting ideal Fitt $\left(I_{2}\right)$ of the quotient module of $E$ given by the $A$-submodule $I_{2}=\left\{\xi(x)-\xi^{\prime}(x) \mid x \in R\right\}$. It then follows from Lemma 2.2 that assertions (i) and (ii) are represented by the quotient algebras $A / \operatorname{Fitt}\left(I_{1}\right)$ and $A / \operatorname{Fitt}\left(I_{2}\right)$, respectively.

2.8. Algebras. By an $A$-algebra $E$, with $E$ not necessarily commutative, we mean a unital ring homomorphism $c: A \longrightarrow E$ from a commutative ring $A$ to an associative, unital ring $E$ where the image $c(A)$ is contained in the center of $E$ [Bou98].

Corollary 2.9. Let $\xi: R \longrightarrow E$ be an A-algebra homomorphism between two not necessarily commutative $A$-algebras. Assume that $E$ is finitely generated and projective as an $A$-module.

(i) Let $I \subseteq R$ be a two-sided ideal. Then $\xi$ factoring through the quotient map $R \longrightarrow R / I$ is a closed condition on $A$.

(ii) Let $\xi^{\prime}: R \longrightarrow E$ be another $A$-algebra homomorphism. Then $\xi$ being equal to $\xi^{\prime}$ is a closed condition on $A$.

Proof. In both cases the question is whether a submodule of $E$ is zero or not. The statement therefore follows from the proposition.

Proposition 2.10. Let $\xi_{i}: R_{i} \longrightarrow E$ be two $A$-algebra homomorphisms between not necessarily commutative $A$-algebras $(i=1,2)$. Assume that $E$ is finitely generated and projective as an $A$-module. Then the condition that $\xi_{1}$ commute with $\xi_{2}$ is a closed condition on $A$. In particular, if $R_{1}$ and $R_{2}$ are commutative, then the two $A$-algebra homomorphisms $\xi_{i}: R_{i} \longrightarrow E$ factoring through $R_{1} \otimes_{A} R_{2}$ is a closed condition on $A$.

Proof. We consider the Fitting ideal Fitt $(I)$, where $I \subseteq E$ is the $A$-submodule

$$
I=\left\{\xi_{1}(x) \xi_{2}(y)-\xi_{2}(y) \xi_{1}(x) \mid x \in R_{1}, y \in R_{2}\right\} .
$$

Then $\xi_{1}$ commutes with $\xi_{2}$ if the module $I$ is the zero module. Hence, by Lemma 2.2 the quotient $A / \operatorname{Fitt}(I)$ represents this condition. That two $A$-algebra homomorphisms $\xi_{i}: R_{i} \longrightarrow E(i=1,2)$ commute is, by the universal property of the tensor product, equivalent to having an $A$-algebra homomorphism $R_{1} \otimes_{A} R_{2} \longrightarrow E$.

\section{Parametrizing algebra homomorphisms}

3.1. Preliminaries. If $V$ is an $A$-module, we let $\mathrm{S}_{A}(V)$ denote the symmetric quotient algebra of the tensor algebra $\mathrm{T}_{A}(V)=\oplus_{n \geqslant 0} V^{\otimes^{n}}$. 


\section{R. M. SkJELnes}

Let $A \longrightarrow R$ and $A \longrightarrow E$ be two, not necessarily commutative, $A$-algebras. We consider the functor $\underline{\operatorname{Hom}}_{A}(R, E)$ that to each commutative $A$-algebra $A^{\prime}$ assigns the set

$$
\operatorname{Hom}_{A \text {-alg }}\left(R, E \otimes_{A} A^{\prime}\right) \text {. }
$$

Remark 3.2. Since $E$ is an $A$-algebra, the tensor product $E \otimes_{A} A^{\prime}$ exists and is an $A^{\prime}$-algebra.

Proposition 3.3. Let $A$ be a commutative ring, and let $A \longrightarrow E$ be an $A$-algebra, where $E$ is not necessarily commutative. Assume that $E$ is finitely generated and projective as an $A$-module. For any $A$-module $V$ the $A$-algebra $\mathrm{S}_{A}\left(V \otimes_{A} E^{\star}\right)$ represents $\underline{\operatorname{Hom}}_{A}(R, E)$, with $R=\mathrm{T}_{A}(V)$.

Proof. An $A$-algebra homomorphism $u_{A^{\prime}}: \mathrm{T}_{A}(V) \longrightarrow E \otimes_{A} A^{\prime}$ is determined by an $A^{\prime}$-linear map $u_{1}: V \otimes_{A} A^{\prime} \longrightarrow E \otimes_{A} A^{\prime}$. Since $E$ is finitely generated and projective, the $A^{\prime}$-linear map $u_{1}$ is equivalent to an $A^{\prime}$-linear map $\varphi_{1}: V \otimes_{A} A^{\prime} \otimes_{A^{\prime}}\left(E \otimes_{A} A^{\prime}\right)^{\star} \longrightarrow A^{\prime}$. Moreover, the canonical map

$$
\operatorname{Hom}_{A}(E, A) \otimes_{A} A^{\prime} \longrightarrow \operatorname{Hom}_{A^{\prime}}\left(E \otimes_{A} A^{\prime}, A^{\prime}\right)
$$

is an isomorphism [Bou98, 4.3. Proposition 7]. Therefore $\varphi_{1}$ corresponds to an $A$-algebra homomorphism $\varphi: \mathrm{S}_{A}\left(V \otimes_{A} E^{\star}\right) \longrightarrow A^{\prime}$. See, for example, [Die62] or [Bou98].

Corollary 3.4. Let $A \longrightarrow R$ and $A \longrightarrow E$ be two, not necessarily commutative, $A$-algebras. Assume that $E$ is finitely generated and projective as an $A$-module. Then $\underline{\operatorname{Hom}}_{A}(R, E)$ is representable.

Proof. Write $R=\mathrm{T}_{A}(V) / I$ for some $A$-module $V$ and some two-sided ideal $I \subseteq \mathrm{T}_{A}(V)$. By Proposition 3.3 the functor $\underline{\operatorname{Hom}}_{A}\left(\mathrm{~T}_{A}(V), E\right)$ is representable by $H=\mathrm{S}_{A}\left(V \otimes_{A} E^{\star}\right)$. Let $\mu: \mathrm{T}_{A}(V) \otimes_{A} H \longrightarrow E \otimes_{A} H$ denote the universal map. We are interested in describing those $A$ algebra maps $H \longrightarrow A^{\prime}$ that factor through $R$. The result now follows by applying Corollary 2.9(i) to the $H$-algebra $\mu$ (where the objects in the category are $H$-algebras and the morphisms are $H$-algebra homomorphisms that also are $A$-linear).

3.5. Non-commutative Weil restrictions. Let $g: A \longrightarrow B$ be a homomorphism of commutative rings. Let $f: B \longrightarrow R$ and $\mu: B \longrightarrow E$ be two $A$-algebra homomorphisms, where $R$ and $E$ are not necessarily commutative. We fix the following data:

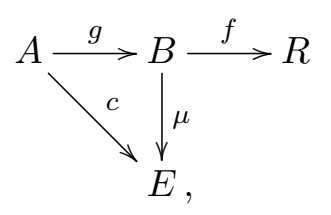

where $c=\mu \circ g$.

Definition 3.6. Fixing the data as above, we consider the functor $\underline{\operatorname{Hom}}_{B / A}(R, E)$ from the category of commutative $A$-algebras to Sets that to any $A$-algebra $A^{\prime}$ assigns the set of $A$-algebra homomorphisms $\xi: R \longrightarrow E \otimes_{A} A^{\prime}$ that fit into the commutative diagram

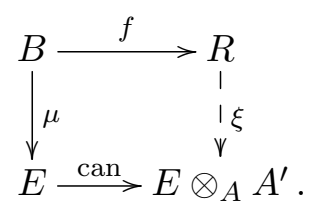

Remark 3.7. If $E$ and $R$ were commutative, then the $A^{\prime}$-valued points $\xi$ of $\underline{\operatorname{Hom}}_{B / A}(R, E)$ would simply have been $B$-algebra homomorphisms. It is important for applications that we have in mind that we do not assume that $\mu: B \longrightarrow E$ is a $B$-algebra. 


\section{WeIL RESTRICTION AND THE QUOT SCHEME}

Remark 3.8. Let $A^{\prime}$ be an $A$-algebra. Having an $A$-algebra homomorphism $\xi: R \longrightarrow E \otimes_{A} A^{\prime}$ is the same as having an $A^{\prime}$-algebra homomorphism $\xi^{\prime}: R \otimes_{A} A^{\prime} \longrightarrow E \otimes_{A} A^{\prime}$. Thus having an $A^{\prime}$-valued point $\xi$ of $\underline{\operatorname{Hom}}_{B / A}(R, E)$ is the same as having an $A^{\prime}$-algebra homomorphism $\xi^{\prime}$ that fits into the commutative diagram

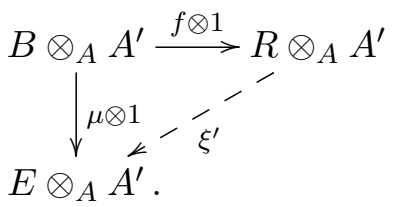

Remark 3.9. When $A=B$, the functor $\underline{\operatorname{Hom}}_{A / A}(R, E)$ is simply the functor $\underline{\operatorname{Hom}}_{A}(R, E)$ discussed in (3.1).

Remark 3.10. If $R$ and $E$ are commutative and $E=B$, then the functor $\underline{\operatorname{Hom}}_{B / A}(R, B)$ is by definition the Weil restriction $\mathfrak{R}_{B / A}(R)$; see, for example, [BLR90].

Proposition 3.11. Let $A \longrightarrow B$ be a homomorphism of commutative rings. Let $A \longrightarrow D$ and $\mu: B \longrightarrow E$ be $A$-algebra homomorphisms, where $E$ is finitely generated and projective as an $A$-module. Then $\underline{\operatorname{Hom}}_{B / A}\left(B \otimes_{A} D, E\right)$ is representable.

Proof. By Lemma 3.4 the functor $\underline{\operatorname{Hom}}_{A}(D, E)$ is representable. Let $H$ be the representing object, and let $u: D \otimes_{A} H \longrightarrow E \otimes_{A} H$ be the universal map. Let $\mu \otimes 1: B \otimes_{A} H \longrightarrow E \otimes_{A} H$ denote the induced map we get from the fixed $A$-algebra homomorphism $\mu: B \longrightarrow E$. By Proposition 2.10 there is a quotient algebra $H \longrightarrow H / I$ representing the closed condition that $\mu \otimes 1$ and $u$ commute. The restrictions of the two maps to $H / I$ factor as

$$
\mu \otimes u: B \otimes_{A} D \otimes_{A} H / I \longrightarrow E \otimes_{A} H / I .
$$

It follows that $H / I$ represents $\underline{\operatorname{Hom}}_{B / A}\left(B \otimes_{A} D, E\right)$.

Corollary 3.12. Let $A \longrightarrow B \longrightarrow R$ be homomorphisms of commutative rings, and let $\mu: B \longrightarrow E$ be an $A$-algebra homomorphism, where the $A$-algebra $E$ is not necessarily commutative, but is finitely generated and projective as an $A$-module. Then $\underline{\operatorname{Hom}}_{B / A}(R, E)$ is representable.

Proof. Write $R$ as a quotient $\mathrm{T}_{A}(V) \otimes_{A} B / I$ for some $A$-module $V$ and some two-sided ideal $I \subseteq \mathrm{T}_{A}(V) \otimes_{A} B$. By the proposition $\underline{\operatorname{Hom}}_{B / A}\left(\mathrm{~T}_{A}(V) \otimes_{A} B, E\right)$ is representable. Let $H$ denote the representing object, and let $u: \mathrm{T}_{A}(V) \otimes_{A} B \otimes_{A} H \longrightarrow E \otimes_{A} H$ denote the universal element. The result then follows by Corollary 2.9(i).

3.13. Weil restrictions and Fitting ideals. Let $A \longrightarrow B$ be a homomorphism of commutative rings, and assume that $B$ is finitely generated and projective as an $A$-module. Let $V$ be an $A$-module. By Proposition 3.3, the $A$-algebra $\mathrm{S}_{A}\left(V \otimes_{A} B^{\star}\right)$ represents $\underline{\operatorname{Hom}}_{A}\left(\mathrm{~T}_{A}(V), B\right)$. Let

$$
u: \mathrm{T}_{A}(V) \longrightarrow B \otimes_{A} \mathrm{~S}_{A}\left(V \otimes_{A} B^{\star}\right)
$$

denote the universal map. We get by extension of scalars an induced $B$-algebra homomorphism

$$
u_{B}: B \otimes_{A} \mathrm{~T}_{A}(V) \longrightarrow B \otimes_{A} \mathrm{~S}_{A}\left(V \otimes_{A} B^{\star}\right) .
$$

For any ideal $I \subseteq B \otimes_{A} \mathrm{~T}_{A}(V)$, we let $u_{B}(I)$ denote the $\mathrm{S}_{A}\left(V \otimes_{A} B^{\star}\right)$-module generated by the image of $I$. 


\section{R. M. SkJELnes}

Corollary 3.14. Let $g: A \longrightarrow B$ be a homomorphism of commutative rings, where $B$ is finitely generated and projective as an $A$-module. Let $f: B \longrightarrow R$ be homomorphisms of rings. Write $R$ as a quotient $B \otimes_{A} \mathrm{~T}_{A}(V) / I$ of the full tensor algebra, where $V$ is some $A$-module. Then the Weil restriction $\mathfrak{R}_{B / A}(R)=\underline{\operatorname{Hom}}_{B / A}(R, B)$ is represented by the $A$-algebra

$$
\mathrm{S}_{A}\left(V \otimes_{A} B^{\star}\right) / \operatorname{Fitt}\left(u_{B}(I)\right),
$$

where $u_{B}$ is the universal map (3.2).

Proof. By Proposition 3.3 the $A$-algebra $\mathrm{S}_{A}\left(V \otimes_{A} B^{\star}\right)$ represents $\underline{\operatorname{Hom}}_{A}\left(\mathrm{~T}_{A}(V), B\right)$. Since $B$ is commutative, it follows that $\mathrm{S}_{A}\left(V \otimes_{A} B^{\star}\right)$ also represents $\underline{\operatorname{Hom}}_{B / A}\left(B \otimes_{A} \mathrm{~T}_{A}(V), B\right)$. Then, finally, the result follows from Corollary 2.9(i).

Remark 3.15. The defining properties of the full tensor algebra $\mathrm{T}_{A}(V)$ and of the symmetric quotient $\mathrm{S}_{A}(V)$ are well known, and can be found, for example, in [Die62] and [Bou98]. The situation with the Weil restriction as in Corollary 3.14 can be found, for example, in [BLR90, Theorem 7.4].

Example 3.16. We will in this example explicitly describe how to compute the Fitting ideals needed to describe the algebra $\underline{\operatorname{Hom}}_{A}(R, E)$ given a presentation of $R=\mathrm{T}_{A}(V) / I$. Assume that the $A$-algebra $E$ is free as an $A$-module with basis $e_{1}, \ldots, e_{n}$, and let $V$ be a free $A$-module with basis $\left\{t_{i}\right\}_{i \in \mathscr{I}}$ for some set $\mathscr{I}$. For any monomial $f=t_{i_{1}} \otimes \cdots \otimes t_{i_{p}}$ in $\mathrm{T}_{A}(V)$, we consider the element $f^{E}$ in $E \otimes_{A} \mathrm{~S}_{A}\left(V \otimes_{A} E^{\star}\right)$ given as

$$
f^{E}=\left(\sum_{k=1}^{n} e_{k} \otimes\left(t_{i_{1}} \otimes e_{k}^{\star}\right)\right) \cdots\left(\sum_{k=1}^{n} e_{k} \otimes\left(t_{i_{p}} \otimes e_{k}^{\star}\right)\right),
$$

where $e_{1}^{\star}, \ldots, e_{n}^{\star}$ is the dual basis of $E^{\star}$. The element $f^{E}$ equals $u(f)$, where $u$ is the universal map

$$
u: \mathrm{T}_{A}(V) \longrightarrow E \otimes_{A} \mathrm{~S}_{A}\left(V \otimes_{A} E^{\star}\right)
$$

Describing the correspondence given by $u$ for monomials will suffice to describe the correspondence for arbitrary elements. We have a unique decomposition

$$
f^{E}=\sum_{k=1}^{n} e_{k} \otimes f_{k}^{E}
$$

with $f_{k}^{E} \in \mathrm{S}_{A}\left(V \otimes_{A} E^{\star}\right)$ for $k=1, \ldots, n$. If we expand the defining expression (3.3) of $f^{E}$, we get

$$
f^{E}=\sum_{\substack{1 \leqslant k_{i} \leqslant n \\ i=1, \ldots, p}} e_{k_{1}} \cdots e_{k_{p}} \otimes\left(t_{i_{1}} \otimes e_{k_{1}}^{\star}\right) \cdots\left(t_{i_{p}} \otimes e_{k_{p}}^{\star}\right) .
$$

Each monomial expression $e_{k_{1}} \cdots e_{k_{p}}$ in the free $A$-module $E$ can be written $\sum_{j=1}^{n} m^{j}(\underline{k}) e_{j}$ for some $m^{j}(\underline{k}) \in A$ with $j=1, \ldots, n$ and $\underline{k}$ the ordered $p$-tuple $k_{1}, \ldots, k_{p}$. Therefore we get

$$
f^{E}=\sum_{j=1}^{n} e_{j} \otimes\left(\sum_{\substack{1 \leqslant k_{i} \leqslant n \\ i=1, \ldots, p}}\left(t_{i_{1}} \otimes e_{k_{1}}^{\star}\right) \cdots\left(t_{i_{p}} \otimes e_{k_{p}}^{\star}\right) \cdot m^{j}(\underline{k})\right) .
$$

In particular, we have

$$
f_{j}^{E}=\sum_{\substack{1 \leqslant k_{i} \leqslant n \\ i=1, \ldots, p}}\left(t_{i_{1}} \otimes e_{k_{1}}^{\star}\right) \cdots\left(t_{i_{p}} \otimes e_{k_{p}}^{\star}\right) \cdot m^{j}(\underline{k})
$$




\section{WeIL RESTRICTION AND THE QUOT SCHEME}

for each $j=1, \ldots, n$. Thus, if we have a two-sided ideal in $\mathrm{T}_{A}(V)$ generated by an element $f$, then $\mathrm{S}_{A}\left(V \otimes_{A} E^{\star}\right) /\left(f_{1}^{E}, \ldots, f_{n}^{E}\right)$ represents $\underline{\operatorname{Hom}}_{A}\left(\mathrm{~T}_{A}(V) /(f), E\right)$.

\section{Module restrictions}

In this section we will introduce the module restriction, which is the main novelty of this article. From now on, the algebras $A \longrightarrow B \longrightarrow R$ are all assumed to be commutative.

4.1. Module structure. Recall that an $A$-module structure on an Abelian group $M$ is assumed to have a ring homomorphism $\rho: A \longrightarrow \operatorname{End}_{\mathbf{z}}(M)$. The image of $\rho$ will factor through the subring of $A$-linear endomorphisms $\operatorname{End}_{A}(M)$, making $\operatorname{End}_{A}(M)$ an $A$-algebra. The ring $\operatorname{End}_{A}(M)$ is unital and associative, and the image of the canonical map can: $A \longrightarrow \operatorname{End}_{A}(M)$ lies in the center.

4.2. Extension of module structures. Let $g: A \longrightarrow B$ be a homomorphism of rings. If $M$ is an $A$-module, then a $B$-module structure on the set $M$ extending the fixed $A$-module structure is a $B$-module structure on $M$ that is compatible with the $A$-module structure. That is, a $B$-module structure on $M$ extending the $A$-module structure is a ring homomorphism $\mu: B \longrightarrow \operatorname{End}_{A}(M)$ that fits into the commutative diagram

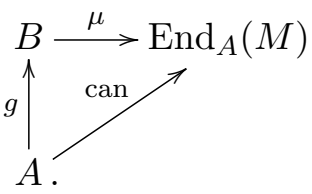

Remark 4.3. If we have an $A$-algebra homomorphism $\mu: B \longrightarrow \operatorname{End}_{A}(M)$, then the map will factor through $\operatorname{End}_{B}(M)$. In particular, $\operatorname{End}_{B}(M)$ is a $B$-algebra, but in general $\operatorname{End}_{A}(M)$ is not a $B$-algebra.

Definition 4.4. Let $g: A \longrightarrow B$ and $f: B \longrightarrow R$ be commutative algebras and homomorphisms, and let $M$ be a $B$-module. We define the functor $\mathscr{M}$ od ${ }_{B \rightarrow R}^{M}$ from the category of $A$-algebras to Sets by assigning to each $A$-algebra $A^{\prime}$ the set

$$
\mathscr{M} \operatorname{od}_{B \rightarrow R}^{M}\left(A^{\prime}\right)=\left\{\begin{array}{l}
R \otimes_{A} A^{\prime} \text {-module structures on } M \otimes_{A} A^{\prime} \text { extending } \\
\text { the fixed } B \otimes_{A} A^{\prime} \text {-module structure on } M \otimes_{A} A^{\prime}
\end{array}\right\} .
$$

We call this functor the module restriction.

Theorem 4.5. Let $g: A \longrightarrow B$ and $f: B \longrightarrow R$ be homomorphisms of commutative rings, and let $M$ be a $B$-module. Assume that $M$, considered as an $A$-module, is projective and finitely generated. Then the functor $\mathscr{M} \operatorname{od}_{B \rightarrow R}^{M}$ is naturally identified with $\underline{\operatorname{Hom}}_{B / A}(R, E)$, where $E=$ $\operatorname{End}_{A}(M)$, and in particular the functor $\mathscr{M} \mathrm{od}_{B \rightarrow R}^{M}$ is representable.

Proof. The $B$-module structure on $M$ is given by an $A$-algebra homomorphism $\mu: B \longrightarrow$ $\operatorname{End}_{A}(M)$. Let $A^{\prime}$ be an $A$-algebra, and let $\xi$ be an $A^{\prime}$-valued point of the module restriction $\mathscr{M} \operatorname{od}_{B \rightarrow R}^{M}$. Then the $A^{\prime}$-valued point $\xi$ is an $A^{\prime}$-algebra homomorphism fitting into the commu- 


\section{R. M. SkJELnES}

tative diagram

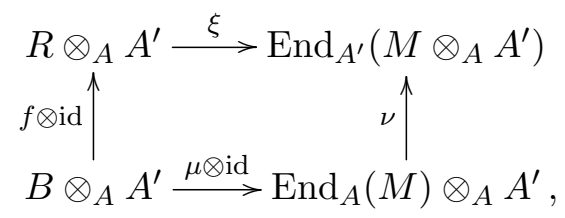

where $\nu$ is the canonical map. Since $M$ is finitely generated and projective, the map $\nu$ is an isomorphism [Bou98, 4.3. Proposition 7]. In other words, we have a natural identification of functors

$$
\mathscr{M} \operatorname{od}_{B \rightarrow R}^{M}=\underline{\operatorname{Hom}}_{B / A}(R, E),
$$

with $E=\operatorname{End}_{A}(M)$. As $M$ is projective and finitely generated, so is $E$, and the statement about representability follows from Corollary 3.14.

\section{Module restriction in a geometric context}

In this section we will set our result on module restrictions in a geometric context.

5.1. Relative rank and support. Let $\mathscr{E}$ be a quasi-coherent sheaf of modules on an algebraic space $X$. If $\mathscr{E}$ is of finite type, then the support is the closed $\operatorname{subspace} \operatorname{Supp}(\mathscr{E})$ of $X$ determined by the annihilator ideal ann $(\mathscr{E})$.

Definition 5.2. Let $g: X \longrightarrow S$ be a morphism of algebraic spaces, and let $\mathscr{E}$ be a sheaf on $X$ that is quasi-coherent and of finite type. We say that $\mathscr{E}$ is finite, flat of relative rank $n$ over $S$ if $\mathscr{E}$ is flat over $S$, the $\operatorname{support} \operatorname{Supp}(\mathscr{E})$ is affine over $S$, and the locally free $\mathscr{O}_{S}$-module $g_{*} \mathscr{E}$ has constant rank $n$.

Proposition 5.3. Let $f: Y \longrightarrow X$ be an affine morphism of $S$-spaces, with $X$ separated. Let $\mathscr{E}$ be a sheaf on $Y$ that is finite, flat of relative rank $n$ over $S$. Then $f_{*} \mathscr{E}$ on $X$ is also finite, flat of relative rank $n$.

Proof. We may assume $S=\operatorname{Spec}(A)$, and then by restricting to the support of $\mathscr{E}$ we may assume $Y=\operatorname{Spec}(B)$. We have by assumption an inclusion $B \longrightarrow \operatorname{End}_{A}(M)$, where $M$ is the $B$-module $M$ corresponding to $\mathscr{E}$. Since $M$ is finitely generated as an $A$-module, $\operatorname{Spec}(B) \longrightarrow \operatorname{Spec}(A)$ is integral. As $X$ is separated, $f: \operatorname{Spec}(B) \longrightarrow X$ is integral [GD61, Proposition 6.1.5]. In particular, $f$ is quasi-compact; we let $X^{\prime}$ denote the scheme-theoretic image of $f$. Then $f: \operatorname{Spec}(B) \longrightarrow X^{\prime}$ is still integral, and surjective; hence $X^{\prime}=\operatorname{Spec}\left(B^{\prime}\right)$ is affine by Chevalley's theorem [Ryd15, Theorem 8.1]. The sheaf $f_{*} \mathscr{E}$ is quasi-coherent and vanishes on the open complement $X \backslash X^{\prime}$. Since $M$ is finitely generated as an $A$-module, it is also finitely generated as a $B^{\prime}$-module. Hence $f_{*} \mathscr{E}$ is of finite type, and the support, given by the annihilator ideal, is a closed subscheme of $X^{\prime}=\operatorname{Spec}(B)$. Thus the support of $f_{*} \mathscr{E}$ is affine, and the result follows.

5.4. The stack of quasi-coherent sheaves. We denote by $\mathscr{C} \mathrm{oh}_{X / S}^{n}$ the stack (see [LMB00] and [Lie06]) of quasi-coherent and finite type sheaves on $X$ that are finite, flat and of relative rank $n$ over $S$. A morphism between two objects $\mathscr{E}$ and $\mathscr{F}$ in $\mathscr{C}$ oh ${ }_{X / S}^{n}(T)$, where $T \longrightarrow S$ is a scheme over $S$, is an $\mathscr{O}_{X \times{ }_{S} T}$-module isomorphism $\varphi: \mathscr{E} \longrightarrow \mathscr{F}$. If $T \longrightarrow S$ is a morphism, then we have an isomorphism of stacks

$$
\mathscr{C} \mathrm{oh}_{X \times{ }_{S} T / T}^{n} \simeq \mathscr{C} \mathrm{oh}_{X / S}^{n} \times_{S} T .
$$


Corollary 5.5. Let $f: Y \longrightarrow X$ be an affine morphism of $S$-spaces with $X$ is separated. Then the push-forward gives a map $f_{*}: \mathscr{C} \mathrm{oh}_{Y / S}^{n} \longrightarrow \mathscr{C} \mathrm{oh}_{X / S}^{n}$.

Proof. Let $T$ be a scheme, let $T \longrightarrow S$ be a morphism, and let $\mathscr{E}$ be an element of $\mathscr{C}$ oh ${ }_{Y / S}^{n}(T)$. To check that $f_{*} \mathscr{E}$ is an element of $\mathscr{C} \mathrm{oh}_{X / S}^{n}(T)$, we may assume that $T$ is an affine scheme, and the result follows.

Theorem 5.6. Let $f: Y \longrightarrow X$ and $g: X \longrightarrow S$ be morphisms of affine schemes $X, Y$ and $S$. Then the push-forward map $f_{*}: \mathscr{C} \mathrm{oh}_{Y / S}^{n} \longrightarrow \mathscr{C} \mathrm{oh}_{X / S}^{n}$ is schematically representable.

Proof. We want to see that for an arbitrary scheme $T$, the fiber product

$$
T \times \mathscr{C o h}_{X / S}^{n} \mathscr{C} \mathrm{oh}_{Y / S}^{n}
$$

is representable by a module restriction. We may assume that $T$ is affine, and by (5.1), that $T=S$. Let $\mathscr{E}$ in $\mathscr{C} \mathrm{oh}_{X / S}^{n}(S)$ be the element corresponding to a given map $S \longrightarrow \mathscr{C} \mathrm{oh}_{X / S}^{n}$. Let $S=\operatorname{Spec}(A), X=\operatorname{Spec}(B)$, and let $\mu: B \longrightarrow \operatorname{End}_{A}(M)$ be the $A$-algebra homomorphism corresponding to the $B$-module structure on $M$, where the module corresponds to the sheaf $\mathscr{E}$. Then $M$ is projective and finitely generated as an $A$-module, and we have by Theorem 4.5 the $A$-algebra $\mathscr{M} \operatorname{od}_{B \rightarrow R}^{M}$, where $Y=\operatorname{Spec}(R)$. We have a natural map

$$
\alpha: \operatorname{Spec}\left(\mathscr{M} \mathrm{od}_{B \rightarrow R}^{M}\right) \longrightarrow S \times \mathscr{C o h}_{X / S}^{n} \mathscr{C} \mathrm{oh}_{Y / S}^{n}
$$

given as follows. Let $u^{\prime}: \operatorname{Spec}\left(A^{\prime}\right) \longrightarrow \operatorname{Spec}\left(\mathscr{M} \operatorname{od}_{B \rightarrow R}^{M}\right)$ be a morphism of affine schemes over $S$, and let $M^{\prime}=M \otimes_{A} A^{\prime}$. By the defining properties of the module restriction $\mathscr{M} \mathrm{od}_{B \rightarrow R}^{M}$, the morphism $u^{\prime}$ corresponds to an $A$-algebra homomorphism $\xi: R \longrightarrow \operatorname{End}_{A^{\prime}}\left(M^{\prime}\right)$ that composed with $B \longrightarrow R$ equals the induced map $B \longrightarrow \operatorname{End}_{A^{\prime}}\left(M^{\prime}\right)$. Then $\alpha\left(u^{\prime}\right)=\left(s^{\prime}, \mathscr{F}_{Y^{\prime}}\right.$, id), where $s^{\prime}: S^{\prime}=\operatorname{Spec}\left(A^{\prime}\right) \longrightarrow S$ is the structure map, and where $\mathscr{F}_{Y^{\prime}}$ is the sheaf on $Y^{\prime}=Y \times_{S} S^{\prime}$ corresponding to the module given by $\xi$. The map $\alpha$ is a monomorphism, and we need to see that it is also essentially surjective.

Let $\left(s^{\prime}, \mathscr{F}, \psi\right)$ be an $S^{\prime}=\operatorname{Spec}\left(A^{\prime}\right)$-valued point of the fiber product (5.2). Then $s^{\prime}: S^{\prime} \longrightarrow S$ is a morphism that when composed with the fixed section of $\mathscr{C} \mathrm{oh}_{X / S}^{n}$ is given by the induced $A^{\prime}$-algebra homomorphism $\mu \otimes 1: B^{\prime} \longrightarrow \operatorname{End}_{A}(M) \otimes_{A} A^{\prime}$, where $B^{\prime}=B \otimes_{A} A^{\prime}$. The sheaf $\mathscr{F}$ is given by an $A^{\prime}$-algebra homomorphism $\xi^{\prime}: R^{\prime} \longrightarrow \operatorname{End}_{A^{\prime}}(N)$ for some projective $A^{\prime}$-module $N$, where $R^{\prime}=R \otimes_{A} A^{\prime}$. Finally, $\psi: M^{\prime} \longrightarrow N$ is an isomorphism of $B^{\prime}$-modules, and in particular an isomorphism of $A^{\prime}$-modules. We then get an induced $A^{\prime}$-algebra isomorphism $\tilde{\psi}: \operatorname{End}_{A^{\prime}}\left(M^{\prime}\right) \longrightarrow$ $\operatorname{End}_{A^{\prime}}(N)$ that respects the $B^{\prime}$-module structures. That is, we have a commutative diagram of $A^{\prime}$-algebras

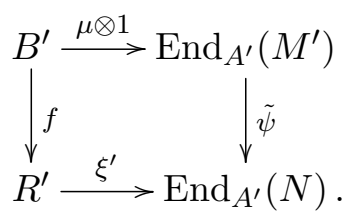

We then have an $A^{\prime}$-algebra homomorphism $\xi=\tilde{\psi}^{-1} \circ \xi^{\prime}$ that extends the map $\mu \otimes 1$. By the defining properties of the module restriction $\mathscr{M} \operatorname{od}_{B \rightarrow R}^{M}$ there exists a unique $u^{\prime}: S^{\prime} \longrightarrow$ $\operatorname{Spec}\left(\mathscr{M} \operatorname{od}_{B \rightarrow R}^{M}\right)$ corresponding to $\xi$. Thus $\alpha\left(u^{\prime}\right)$ is isomorphic to $\left(s^{\prime}, \mathscr{F}, \psi\right)$, and we have shown that $\alpha$ is essentially surjective.

Corollary 5.7. Let $f: Y \longrightarrow X$ be an affine morphism of $S$-spaces, with $X$ separated. Then the push-forward map $f_{*}: \mathscr{C} \mathrm{oh}_{Y / S}^{n} \longrightarrow \mathscr{C}_{\mathrm{oh}}^{n}{ }_{X / S}$ is schematically representable. 


\section{R. M. SkJELnES}

Proof. Let $\mathscr{E}$ be an element of $\mathscr{C} \mathrm{oh}_{X / S}^{n}(T)$, where $T \longrightarrow S$ is a scheme. We may assume $T=S$, and that the base $S$ is affine. We may furthermore replace $X$ with the support of $\operatorname{Supp}(\mathscr{E})=$ $\operatorname{Spec}(B)$, and then replace $Y$ with $\operatorname{Spec}\left(B^{\prime}\right)=Y \times_{S} X$. The result then follows from the theorem.

Lemma 5.8. Let $X \longrightarrow S$ be a separated map of algebraic spaces, and let $f: Y \longrightarrow X$ be an affine morphism. Then the natural morphism

$$
\mathscr{C} \mathrm{oh}_{Y / S}^{n} \times \mathscr{C} \mathrm{oh}_{X / S}^{n} \mathscr{C} \mathrm{oh}_{Y / S}^{n} \longrightarrow \mathscr{C} \mathrm{oh}_{Y / S}^{n} \times{ }_{S} \mathscr{C} \mathrm{oh}_{Y / S}^{n}
$$

is a closed immersion.

Proof. We may assume that $S=\operatorname{Spec}(A)$ is affine. By (5.1) it suffices to check closedness for $S$-valued points. Let $\mathscr{E}_{1}$ and $\mathscr{E}_{2}$ be two $S$-valued points of $\mathscr{C} \mathrm{oh}_{Y / S}^{n}$ such that $h_{*} \mathscr{E}_{1}=h_{*} \mathscr{E}_{2}$, where $h: Y \longrightarrow S$ is the structure map. Let $M$ be the $A$-module of global sections $\Gamma\left(S, h_{*} \mathscr{E}_{1}\right)$. Let $\operatorname{Spec}(R) \subseteq Y$ denote the support of $\mathscr{E}_{1}$. Then the restriction of the sheaf $\mathscr{E}_{i}(i=1,2)$ to $\operatorname{Spec}(R)$ is given by an $A$-algebra homomorphism $\xi_{i}: R \longrightarrow \operatorname{End}_{A}(M)$. The support of $f_{*} \mathscr{E}_{1}$ on $X$ is affine (Proposition 5.3), say given as $\operatorname{Spec}(B) \subseteq X$. Denote by $\varphi: B \longrightarrow R$ the induced $A$-algebra homomorphism. Then the restriction of $f_{*} \mathscr{E}_{i}$ to $\operatorname{Spec}(B)$ is given by the composite $A$-algebra homomorphism $(i=1,2)$

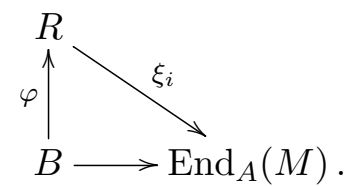

By Corollary 2.9 the equality of the two maps $\xi_{1} \circ \varphi$ and $\xi_{2} \circ \varphi$ is a closed condition on $A$. Applying the same argument to the support $\operatorname{Spec}\left(R^{\prime}\right) \subseteq Y$ of $\mathscr{E} 2$ gives us a closed condition on $S$ for where the two sheaves $f_{*} \mathscr{E}_{1}$ and $f_{*} \mathscr{E}_{2}$ are equal. We have then shown that for an $S$-valued point of the fiber product on the right in the statement, factoring through the fiber product on the left is a closed condition on $S$.

\section{Weil restriction revisited}

We will in this section define an open subfunctor of the module restriction that inherits properties as étaleness. This open subfunctor is important for the application we give in the last section.

Proposition 6.1. Let $f: B \longrightarrow R$ be a homomorphism of commutative $A$-algebras, and let $\mathfrak{R}_{B / A}(R)=\underline{\operatorname{Hom}}_{B / A}(R, B)$ denote the Weil restriction functor.

(i) If $f: B \longrightarrow R$ is of finite presentation, then $\mathfrak{R}_{B / A}(R)$ is of finite presentation.

(ii) If $f: B \longrightarrow R$ satisfies the infinitesimal lifting property for étaleness or for smoothness, then the functor $\mathfrak{R}_{B / A}(R)$ satisfies the corresponding infinitesimal lifting property.

Proof. We will use the functorial characterization [GD66, Corollaire (8.14.2.2)] to prove the first assertion. If we have a directed system of $A$-algebras (and $A$-algebra homomorphisms) $\left\{A_{\alpha}\right\}_{\alpha \in \mathscr{A}}$, then we obtain an induced injective map

$$
\underset{\lim }{\longrightarrow} \Re_{B / A}(R)\left(A_{\alpha}\right) \longrightarrow \Re_{B / A}(R)\left(\lim _{\longrightarrow}\left(A_{\alpha}\right)\right) .
$$

We need to see that this map (6.1) is a bijection; that is, we need to check surjectivity. Let $\lim _{\longrightarrow} A_{\alpha}=A^{\prime}$, and let $\xi^{\prime} \in \mathfrak{R}_{B / A}(R)\left(A^{\prime}\right)$. By definition $f \circ \xi^{\prime}$ equals the canonical map $B \longrightarrow$ 


\section{WeIL RESTRICTION AND THE QUOT SCHEME}

$B \otimes_{A} A^{\prime}$. Hence $\xi^{\prime}$ is a $B$-algebra homomorphism. As $\lim _{\longrightarrow}\left(B \otimes_{A} A_{\alpha}\right)=B \otimes_{A} A^{\prime}$, and as $f: B \longrightarrow R$ is of finite presentation, we have a bijection

$$
\underset{\lim }{\longrightarrow}\left(\operatorname{Hom}_{B \text {-alg }}\left(R, B \otimes_{A} A_{\alpha}\right)\right) \longrightarrow \operatorname{Hom}_{B \text {-alg }}\left(R, B \otimes_{A} A^{\prime}\right) \text {. }
$$

Consequently $\xi^{\prime}=\left\{\xi_{\alpha}\right\}$ is a sequence of $B$-algebra homomorphisms $\xi_{\alpha} \in \mathfrak{R}_{B / A}(R)\left(A_{\alpha}\right)$. Thus the map (6.1) is a bijection, and $\mathfrak{R}_{B / A}(R)$ is of finite presentation. To check the two remaining assertions, let $A^{\prime}$ be an $A$-algebra, and let $N \subseteq A^{\prime}$ be a nilpotent ideal. Let $\xi_{N}$ be an $A^{\prime} / N$-valued point of $\mathfrak{R}_{B / A}(R)$. We then obtain the following commutative diagram:

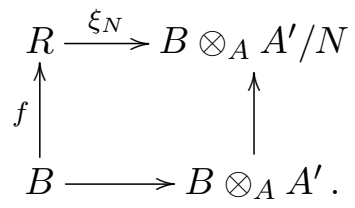

Now, as $N \subseteq A^{\prime}$ is nilpotent, the kernel of the canonical map

$$
B \otimes_{A} A^{\prime} \longrightarrow B \otimes_{A} A^{\prime} / N=B / N B
$$

is nilpotent. Then if $f: B \longrightarrow R$ has an infinitesimal lifting property, we obtain a lifting $\xi^{\prime}: R \longrightarrow B \otimes_{A} A^{\prime}$ of $\xi_{N}$. Then $\xi^{\prime}$ is an $A^{\prime}$-valued point of $\mathfrak{R}_{B / A}(R)$, and we have proved the last assertions.

Remark 6.2. It is well known that the Weil restriction $\mathfrak{R}_{B / A}(R)$ inherits properties such as étaleness or smoothness if $B \longrightarrow R$ is étale or smooth, respectively. In [BLR90], for example, these and other properties are shown for the Weil restriction, however with some assumptions that do not apply in our context.

Example 6.3. We give here an example showing that if $B \longrightarrow R$ is étale, then $\underline{\operatorname{Hom}}_{B / A}(R, E)$ will not necessarily satisfy the infinitesimal lifting property. That fact was pointed out to us by Laksov, who thereby corrected an error we had in a earlier version of this article. Consider first the matrices

$$
x=\left[\begin{array}{ll}
0 & \epsilon \\
0 & 0
\end{array}\right] \text { and } y=\left[\begin{array}{ll}
a_{1}+a_{2} \epsilon & b_{1}+b_{2} \epsilon \\
c_{1}+c_{2} \epsilon & d_{1}+d_{2} \epsilon
\end{array}\right],
$$

where the entries of the matrices are in some ring $A$, and where $\epsilon$ is a non-zero element such that $\epsilon^{2}=0$. Since

$$
x y=\left[\begin{array}{cc}
a_{1} \epsilon & b_{1} \epsilon \\
0 & 0
\end{array}\right] \text { and } y x=\left[\begin{array}{ll}
0 & b_{1} \epsilon \\
0 & c_{1} \epsilon
\end{array}\right],
$$

these matrices do not in general commute. However, when we set $\epsilon=0$, the matrix $x$ becomes the zero matrix, and the reduced matrices clearly commute. Therefore we have the following. Let $A=k[\epsilon] /\left(\epsilon^{2}\right)$ over a field $k$. Let $B=A[X]$ denote the polynomial ring in the variable $X$ over $A$, and let $M=A \oplus A$. The matrix $x$ gives a $B$-module structure on $M$ by sending the variable $X$ to the matrix $x$. Thus we have an $A$-algebra homomorphism $\mu: B \longrightarrow \operatorname{End}_{A}(A \oplus A)=E$. We let $R=A[X, Y] /\left(Y^{2}-1\right)$, which is étale over $B$ when the characteristic of $k$ is different from two. We let furthermore $A^{\prime}=A$, and consider the nilpotent ideal $N=(\epsilon) \subseteq A$. We then have the following commutative diagram:

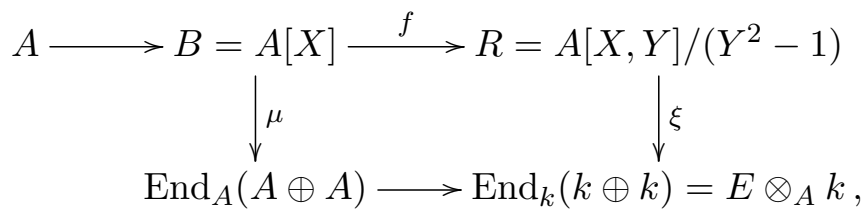




\section{R. M. SkJELnes}

where $\xi: R \longrightarrow \operatorname{End}_{k}(k \oplus k)$ is determined by sending $X$ to 0 and sending $Y$ to the endomorphism given by the matrix $\bar{y}=\left[\begin{array}{rr}0 & -1 \\ -1 & 0\end{array}\right]$. Any lifting of $\bar{y}$ to an element in $\operatorname{End}_{A}(A \oplus A)$ is of the form

$$
y=\left[\begin{array}{cc}
a_{2} \epsilon & -1+b_{2} \epsilon \\
-1+c_{2} \epsilon & d_{2} \epsilon
\end{array}\right],
$$

with elements $a_{2}, b_{2}, c_{2}, d_{2}$ in $k$. From the considerations above no such lifting will commute with the matrix $x$. Therefore there exist no $B$-algebra homomorphisms $\tilde{\xi}: R \longrightarrow \operatorname{End}_{A}(A \oplus A)$ that extend $\xi$. Thus, even if $f: B \longrightarrow R$ is étale, the $A$-algebra $\underline{\operatorname{Hom}}_{B / A}(R, E)$ is not necessarily étale.

Example 6.4. Our next example shows that even if $B \longrightarrow R$ is of finite presentation, the $A$ algebra representing $\underline{\operatorname{Hom}}_{B / A}(R, E)$ is not of finite presentation. It follows, though, from the constructions given in the proofs (of the representability) that if $B \longrightarrow R$ is of finite type, then the $A$-algebra $\underline{\operatorname{Hom}}_{B / A}(R, E)$ is of finite type.

Let $A=k\left[w_{i}\right]_{i \geqslant 0}$ be the polynomial ring in a countable number of variables $w_{1}, w_{2}, \ldots$ over some ring $k$. Let $M=A \oplus A$ be the free $A$-module of rank two. From the polynomial ring in one variable $A[T]$ over $A$, we obtain an $A[T]$-module structure on $M$ by sending $T$ to the matrix

$$
t=\left[\begin{array}{ll}
0 & 1 \\
1 & 0
\end{array}\right]
$$

Let $B=A\left[X_{i}\right]_{i \geqslant 1}$ be the polynomial ring in the variables $X_{1}, X_{2}, \ldots$ over $A$. For each $i$ we consider the matrix

$$
x_{i}=\left[\begin{array}{cc}
w_{i} & 0 \\
0 & w_{i+1}
\end{array}\right] .
$$

Since the diagonal matrices commute, we get a $B$-module structure on $M$ by sending the variable $X_{i}$ to the matrix $x_{i}$. One checks that the two $A$-algebra homomorphisms $\mu: B \longrightarrow E=$ $\operatorname{End}_{A}(M)$ and $u: A[T] \longrightarrow E$ commute if and only if $w_{i}=w_{i+1}$ for all $i \geqslant 1$. Thus, the closed condition on $A$ for where the two maps $\mu$ and $u$ commute is given by $A /\left(w_{i}-w_{i+1}\right)_{i \geqslant 1}$, which is not of finite presentation. As finite presentation is preserved under specialization, the $A$-algebra $\underline{\operatorname{Hom}}_{B / A}\left(B \otimes_{A} A[T], E\right)$ cannot be of finite presentation either.

6.5. Isomorphic image functor. Let $f: B \longrightarrow R$ be a homomorphism of commutative $A$-algebras, and let $\mu: B \longrightarrow E$ be a homomorphism of $A$-algebras, where $E$ is not necessarily commutative. We will define a subfunctor

$$
\underline{\operatorname{Im}}_{B=R}^{E} \subseteq \underline{\operatorname{Hom}}_{B / A}(R, E) .
$$

Recall that an $A^{\prime}$-valued point of $\underline{\operatorname{Hom}}_{B / A}(R, E)$ is an $A^{\prime}$-algebra homomorphism $\xi^{\prime}$ that fits into the following commutative diagram:

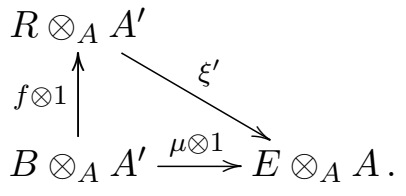

For any $A$-algebra $A^{\prime}$, we let

$$
\underline{\operatorname{Im}}_{B=R}^{E}\left(A^{\prime}\right)=\left\{\xi^{\prime} \in \underline{\operatorname{Hom}}_{B / A}(R, E)\left(A^{\prime}\right) \mid \mu \otimes 1=\xi^{\prime} \circ f \otimes 1\right\} .
$$

The functor $\underline{\operatorname{Im}}_{B=R}^{E}$ parametrizes $A$-algebra homomorphisms $R \longrightarrow E$ such that the image of $R$ equals the image of $B \longrightarrow E$. 


\section{WeIL RESTRICTION AND THE QUOT SCHEME}

Proposition 6.6. Let $A \longrightarrow B \longrightarrow R$ be homomorphisms of commutative rings, and let $\mu: B \longrightarrow E$ be an $A$-algebra homomorphism with $E$ not necessarily commutative. Assume that $E$ is finitely generated and projective as an $A$-module and that $B \longrightarrow R$ is of finite type. Then the functor $\underline{\operatorname{Im}}_{B=R}^{E}$ is representable by an open subscheme of $\underline{\operatorname{Hom}}_{B / A}(R, E)$.

Proof. By Corollary 3.12 the functor $\underline{\operatorname{Hom}}_{B / A}(R, E)$ is representable; let $H$ be the $A$-algebra representing the functor. Let $\xi: R \otimes_{A} H \longrightarrow E \otimes_{A} H$ denote the universal element of $\underline{\operatorname{Hom}}_{B / A}(R, E)$, and let $\operatorname{Im}(\xi) \subseteq E \otimes_{A} H$ denote the image of $\xi$. Let furthermore $B_{H}$ denote the image of $\mu \otimes 1: B \otimes_{A} H \longrightarrow E \otimes_{A} H$. We have the inclusion of $B_{H}$-modules $B_{H} \subseteq \operatorname{Im}(\xi) \subseteq E \otimes_{A} H$. Any element $x \in E \otimes_{A} H$ gives, by multiplication, an $H$-linear endomorphism on $E \otimes_{A} H$. The Cayley-Hamilton theorem guarantees that the element $x$ will satisfy its characteristic polynomial, and consequently that $x$ is integral over $H$. From this we deduce the following two consequences. First, since $R$ is finite type over $B$, the image $\operatorname{Im}(\xi)$ is a finitely generated $B_{H}$-module. In particular, the quotient $B_{H}$-module $\operatorname{Im}(\xi) / B_{H}$ has closed support $Z \subseteq \operatorname{Spec}\left(B_{H}\right)$ given by the annihilator ideal $\operatorname{ann}_{B_{H}}\left(\operatorname{Im}(\xi) / B_{H}\right)$. Second, as $B_{H}$ is integral over $H$, the corresponding morphism $g: \operatorname{Spec}\left(B_{H}\right) \longrightarrow \operatorname{Spec}(H)$ is closed. Thus $g(Z)$ is the closed subscheme given by the ideal $\operatorname{ann}_{B_{H}}\left(\operatorname{Im}(\xi) / B_{H}\right) \cap H$. And as $g^{-1}(g(Z))=Z$, it is clear that the open subscheme $U=\operatorname{Spec}(H) \backslash g(Z)$ represents $\underline{\operatorname{Im}}_{B=R}^{E}$.

Remark 6.7. Similar results can be found in [Ryd08].

6.8. Properties of the isomorphic image functor. We keep the notation introduced above 6.5 and assume that the $A$-algebra $E$ is finitely generated and projective as an $A$-module. Let $H$ be the $A$-algebra representing $\underline{\operatorname{Hom}}_{B / A}(R, E)$, and let $\xi: R \otimes_{A} H \longrightarrow E \otimes_{A} H$ denote the universal element. We then have the following commutative diagram:

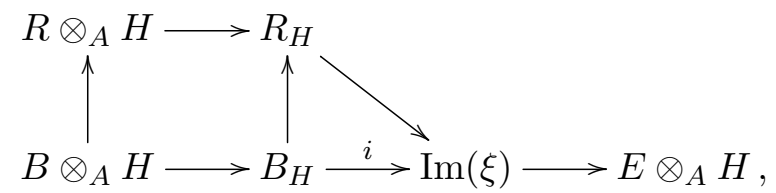

where $B_{H}$ is the image of $\mu \otimes 1: B \otimes_{A} H \longrightarrow E \otimes_{A} H$, the map $i$ is the natural inclusion and $R_{H}=R \otimes_{B} B_{H}$.

Proposition 6.9. Let $A \longrightarrow B \longrightarrow R$ be homomorphisms of commutative rings, and let $\mu: B \longrightarrow E$ be an $A$-algebra homomorphism, with $E$ not necessarily commutative. Assume that $E$ is finitely generated and projective as an $A$-module and that $B \longrightarrow R$ is of finite type. Let $H$ be the $A$-algebra representing $\underline{\operatorname{Hom}}_{B / A}(R, E)$. Let $B_{H}$ denote the image of the composite map $\mu \otimes 1: B \otimes_{A} H \longrightarrow E \otimes_{A} H$, and let $R_{H}=R \otimes_{B} B_{H}$. Then the functor $\underline{I m}_{B=R}^{E}$ equals the Weil restriction $\mathfrak{R}_{B_{H} / A}\left(R_{H}\right)$. In particular, the Weil restriction $\mathfrak{R}_{B_{H} / A}\left(R_{H}\right)$ is representable by a scheme.

Proof. By the finite type assumption on $B \longrightarrow R$, by Proposition 6.6 the functor $\underline{I m}_{B=R}^{E}$ is represented by an open subscheme $U \subseteq \operatorname{Spec}(H)$. When we restrict diagram (6.2) to the open subscheme $U \subseteq \operatorname{Spec}(H)$, by definition the map of $\mathscr{O}_{U}$-modules

$$
i_{\mid U}: B_{H \mid U} \longrightarrow \operatorname{Im}(\xi)_{\mid U}
$$

is surjective. As $U \subseteq \operatorname{Spec}(H)$ is an open immersion, and in particular a flat map that preserves injectivity, the restriction morphism (6.3) is an isomorphism. Composition of the restriction of 


\section{R. M. SkJELnES}

the universal map

$$
\xi_{\mid U}: R \otimes_{A} H_{\mid U} \longrightarrow E \otimes_{A} H_{\mid U}
$$

with the inverse of (6.3) induces a map $R_{H \mid U} \longrightarrow B_{H \mid U}$, in other words, a $U$-valued point of the Weil restriction $\mathfrak{R}_{B_{H} / A}\left(R_{H}\right)$. And conversely, any $A^{\prime}$-valued point of the Weil restriction composed with the induced map $i \otimes 1: B_{H} \otimes_{A} A^{\prime} \longrightarrow E \otimes_{A} A^{\prime}$ is an $A^{\prime}$-valued point of $\underline{\operatorname{Im}}_{B=R}^{E}$.

Remark 6.10. Note that the $A$-algebra $B_{H}$ is not assumed to be finitely generated or projective as an $A$-module. Those conditions are required for the representability of the Weil restriction, for example in [BLR90].

Corollary 6.11. Let $A \longrightarrow B \longrightarrow R$ be homomorphisms of commutative rings, and let $\mu: B \longrightarrow E$ be an $A$-algebra homomorphism, with $E$ not necessarily commutative. Assume that $E$ is finitely generated and projective as an $A$-module and that $B \longrightarrow R$ is étale (respectively, smooth). Then the scheme representing the functor $\underline{\operatorname{Im}}_{B=R}^{E}$ is étale (respectively, smooth).

Proof. If $f: B \longrightarrow R$ is étale or smooth, then in particular it is of finite type. Hence, by Proposition 6.6, the functor $\underline{\operatorname{Im}}_{B=R}^{E}$ is representable by a scheme. Moreover, by Proposition 6.9, the image $\underline{\operatorname{Im}}_{B=R}^{E}$ equals the Weil restriction $\mathfrak{R}_{B_{H} / A}\left(R_{H}\right)$, where we use the notation of Proposition 6.9. As $f: B \longrightarrow R$ is étale (respectively, smooth), by base change $B_{H} \longrightarrow R_{H}$ is étale (respectively, smooth). The result then follows by Proposition 6.1.

Corollary 6.12. Let $f: B \longrightarrow R$ be an $A$-algebra homomorphism. Let $M$ be a $B$-module, and let $E=\operatorname{End}_{A}(M)$. Assume that $M$ is finitely generated and projective as an $A$-module. Then for any $A$-algebra $A^{\prime}$, the $A^{\prime}$-valued points of $\underline{\operatorname{Im}}_{B=R}^{E}$ correspond to $R^{\prime}=R \otimes_{A} A^{\prime}$-module structures on $M \otimes_{A} A^{\prime}$ extending the fixed $B^{\prime}=B \otimes_{A} A^{\prime}$-module structure and such that the induced map of supports

is an isomorphism.

$$
B^{\prime} / \operatorname{ann}_{B^{\prime}}\left(M \otimes_{A} A^{\prime}\right) \longrightarrow R^{\prime} / \operatorname{ann}_{R^{\prime}}\left(M \otimes_{A} A^{\prime}\right)
$$

Proof. As $M$ is a projective and finitely generated $A$-module, $\operatorname{End}_{A}(M) \otimes_{A} A^{\prime}=\operatorname{End}_{A^{\prime}}\left(M \otimes_{A} A^{\prime}\right)$ for any $A$-algebra $A^{\prime}$. Then, for such an $A^{\prime}$, the kernel of $\mu \otimes 1: B \otimes_{A} A^{\prime} \longrightarrow E \otimes_{A} A^{\prime}$ is the annihilator ideal. Thus, $B^{\prime} / \operatorname{ann}_{B^{\prime}}\left(M \otimes_{A} A^{\prime}\right)$, with $B^{\prime}=B \otimes_{A} A^{\prime}$, is the image of $\mu \otimes 1$. If $\xi^{\prime}$ is an $A^{\prime}$-valued point of $\underline{\operatorname{Im}}_{B=R}^{E}$, then by definition

$$
B^{\prime} / \operatorname{ann}_{B^{\prime}}\left(M \otimes_{A} A^{\prime}\right)=\operatorname{Im}(\mu \otimes 1)=\operatorname{Im}\left(\xi^{\prime}\right)=R^{\prime} / \operatorname{ann}_{R^{\prime}}\left(M \otimes_{A} A^{\prime}\right),
$$

where $R^{\prime}=R \otimes_{A} A^{\prime}$.

6.13. Situation with commutative rings. We will end this section by showing that when the module $M$ actually is an algebra, our functors specialize to the ordinary Weil restriction.

Proposition 6.14. Let $A \longrightarrow B \longrightarrow R$ be homomorphisms of commutative rings. Let $E=$ $\operatorname{End}_{A}(B)$, and let $\mu: B \longrightarrow E$ denote the canonical map. We have the equality of functors

$$
\mathfrak{R}_{B / A}(R)=\underline{\operatorname{Im}}_{B=R}^{E}=\underline{\operatorname{Hom}}_{B / A}(R, E) .
$$

Proof. We first note that the map $\mu: B \longrightarrow \operatorname{End}_{A}(B)$ is universally injective. Let $A^{\prime}$ be an $A$-algebra. We have natural maps

$$
B \otimes_{A} A^{\prime} \stackrel{\mu \otimes 1}{\longrightarrow} \operatorname{End}_{A}(B) \otimes_{A} A^{\prime} \longrightarrow \operatorname{End}_{A^{\prime}}\left(B \otimes_{A} A^{\prime}\right),
$$




\section{WeIL RESTRICTION AND THE QUOT SCHEME}

where the composite is the canonical map $\mu^{\prime}: B^{\prime} \longrightarrow \operatorname{End}_{A^{\prime}}\left(B^{\prime}\right)$ with $B^{\prime}=B \otimes_{A} A^{\prime}$. The map $\mu^{\prime}$ is injective, and it follows that $B \otimes_{A} A^{\prime} \longrightarrow \operatorname{End}_{A}(B) \otimes_{A} A^{\prime}$ is injective for all $A$-algebras $A^{\prime}$.

We then prove the equalities in the proposition. Let $\xi^{\prime}$ be an $A^{\prime}$-valued point of $\underline{\operatorname{Hom}}_{B / A}(R, E)$. It is readily verified that $\xi^{\prime}$ will factor through $\operatorname{Im}(\mu \otimes 1)$. The equality of $\underline{\operatorname{Im}}_{B=R}^{E}=\underline{\operatorname{Hom}}_{B / A}(R, E)$ then follows from the definitions.

Since $\mu \otimes 1$ is furthermore injective, we may identify $B \otimes_{A} A^{\prime}$ with its image in $E \otimes_{A} A^{\prime}$. The map $\xi^{\prime}$ then equals a map $R \otimes_{A} A^{\prime} \longrightarrow B \otimes_{A} A^{\prime}$, that is, an $A^{\prime}$-valued point of the Weil restriction $\mathfrak{R}_{B / A}(R)$. The equality of the two functors $\mathfrak{R}_{B / A}(R)$ and $\underline{\operatorname{Im}}_{B=R}^{E}$ follows.

\section{Representability of the Quot functor}

In this last section we will apply our result to show that the Quot functor of finitely supported quotients is representable.

7.1. The stack of isomorphic supports. Let $f: Y \longrightarrow X$ be a morphism of $S$-spaces, with $X$ separated. For each scheme $T$ and for any element $\mathscr{E}$ in $\mathscr{C}$ oh ${ }_{Y / S}^{n}(T)$ we have an induced map on supports

$$
f_{T \mid}: \operatorname{Supp}(\mathscr{E}) \longrightarrow \operatorname{Supp}\left(f_{*} \mathscr{E}\right)
$$

Let

$$
\mathscr{U}_{Y \rightarrow X} \subset \mathscr{C}_{\mathrm{oh}_{Y / S}}^{n}
$$

denote the substack whose objects are $\mathscr{O}_{Y_{T}}$-modules $\mathscr{E} \in \mathscr{C}$ oh $\mathrm{Y}_{Y / S}(T)$ such that the induced map on supports is a closed immersion.

Proposition 7.2. Let $f: Y \longrightarrow X$ be an $S$-morphism that is affine and of finite type, with $X$ separated. Then the induced map of stacks

$$
\mathscr{U}_{Y \rightarrow X} \longrightarrow \mathscr{C o h}_{Y / S}^{n}
$$

is a representable open immersion. If furthermore $f: Y \longrightarrow X$ is étale (respectively, smooth), then the induced composite map

$$
\mathscr{U}_{Y \rightarrow X} \longrightarrow \mathscr{C}_{\mathrm{oh}_{Y / S}^{n}} \longrightarrow \mathscr{C}_{\mathrm{oh}}^{n}{ }_{X / S}
$$

is étale (respectively, smooth).

Proof. The results follow from Proposition 6.6 and Corollary 6.11.

7.3. The Quot stack. Fix a quasi-coherent sheaf $F_{X}$ on an algebraic space $X \longrightarrow S$. For any $S$-scheme $T$ we let $F_{X_{T}}$ denote the pull-back of $F_{X}$ along the first projection $p_{X}: X \times_{S} T \longrightarrow X$. The $T$-valued points of the Quot stack $\mathfrak{Q u o t}_{F_{X} / S}^{n}$ are all $\mathscr{O}_{X \times_{S} T}$-module morphisms $q: F_{X_{T}} \longrightarrow \mathscr{E}$ from $F_{X_{T}}$ to a quasi-coherent sheaf $\mathscr{E}$ on $X \times_{S} T$, where $\mathscr{E}$ is finite, flat of relative rank $n$ over $T$. A morphism between two objects $q: F_{X_{T}} \longrightarrow \mathscr{E}$ and $q^{\prime}: F_{X_{T}} \longrightarrow \mathscr{E}^{\prime}$ is an $\mathscr{O}_{X \times{ }_{S} T}$-module isomorphism $\varphi: \mathscr{E} \longrightarrow \mathscr{E}^{\prime}$ such that $q^{\prime}=\varphi \circ q$.

Remark 7.4. Note that the maps $q: F_{X_{T}} \longrightarrow \mathscr{E}$ are not assumed to be surjective, and in particular the $T$-valued points of the Quot stack $\mathfrak{Q u o t}_{F_{X} / S}^{n}$ are not quotients of $F_{X}$. The definition of the Quot stack is motivated by the definition of the Hilbert stack in [Ryd11] and in [Ols06]. 


\section{R. M. SKJELNES}

7.5. Identification of pull-backs. We will in the remainder of this article return to a particular situation that we describe below. Let $T \longrightarrow S$ be a morphism. Then we have the following Cartesian diagram:

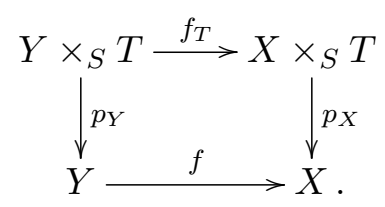

For any sheaf $F_{X}$ on $X$ there is a canonical identification between the two sheaves $f_{T}^{*} F_{X_{T}}=$ $f_{T}^{*} p_{X}^{*} F_{X}$ and $p_{Y}^{*} f^{*} F_{X}$. We will denote both these two sheaves by $F_{Y_{T}}$. We have, furthermore, a natural map

$$
c: \mathfrak{Q u o t}_{F_{X} / S}^{n} \longrightarrow \mathscr{C} \mathrm{oh}_{X / S}^{n}
$$

that takes a $T$-valued point $q: F_{X_{T}} \longrightarrow \mathscr{E}$ of the quotient stack to the sheaf $\mathscr{E}$.

Lemma 7.6. Let $f: Y \longrightarrow X$ be a morphism of $S$-spaces, with $X$ separated. For any quasicoherent sheaf $F_{X}$ on $X$, we have the Cartesian diagram

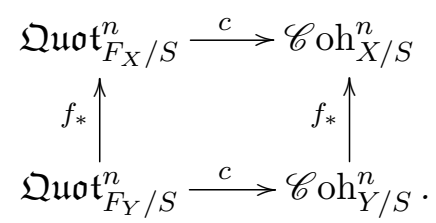

Proof. We first establish the map $f_{*}$ from $\mathfrak{Q u o t}_{F_{Y} / S}^{n}$ to $\mathfrak{Q u o t}_{F_{X} / S}^{n}$. Let $q: F_{Y_{T}} \longrightarrow \mathscr{E}$ be a $T$ valued point of $\mathfrak{Q u o t}_{F_{Y} / S}^{n}$. The canonical map $F_{X_{T}} \longrightarrow f_{T *} f_{T}^{*} F_{X_{T}}$, where we use the notation of (7.2), combined with the identification $f_{T}^{*} F_{X_{T}}=F_{Y_{T}}$, gives the composition

$$
F_{X_{T}} \longrightarrow f_{T *} f_{T}^{*} F_{X_{T}}=f_{T *} F_{Y_{T}} \longrightarrow f_{T *} \mathscr{E} .
$$

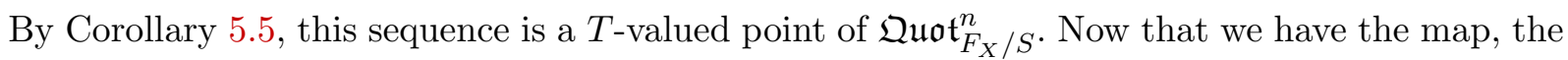
proof is a formal consequence of adjunction.

7.7. The Quot functor. Let $X \longrightarrow S$ be a morphism of algebraic spaces, and let $F_{X}$ be a quasicoherent sheaf on $X$. We define the Quot functor Quot $_{F_{X} / S}^{n}$ as the functor that to each $S$-scheme $T \longrightarrow S$ assigns the set of surjective $\mathscr{O}_{X_{T}}$-module maps $q: F_{X_{T}} \longrightarrow \mathscr{E}$, where $\mathscr{E}$ is finite, flat and of relative rank $n$. Two surjective maps $q: F_{X_{T}} \longrightarrow \mathscr{E}$ and $q^{\prime}: F_{X_{T}} \longrightarrow \mathscr{E}$ are considered equal if their kernels coincide as subsheaves of $F_{X_{T}}$. This definition of $\underline{\text { uot }}_{F_{X} / S}^{n}$ extends the one given by Grothendieck [Gro95, Art69] for projective schemes $X \longrightarrow S$.

Let $q: F_{X_{T}} \longrightarrow \mathscr{E}$ be a $T$-valued point of $\underline{\text { Quot }}_{F_{X} / S}^{n}$. We define

$$
\iota(\mathscr{E})=\mathscr{O}_{X \times{ }_{S} T} / \operatorname{ker} q .
$$

This determines a map $\iota: \underline{\operatorname{Quot}}_{F_{X} / S}^{n} \longrightarrow \mathfrak{Q u o t}_{F_{X}, S}^{n}$.

7.8. Subfunctors of the Quot functor. We will define two subfunctors of the Quot functor, and then in the next lemma relate these subfunctors to the stacks introduced earlier. Let $f: Y \longrightarrow X$ be a morphism of $S$-spaces with $X$ separated, and let $F_{X}$ be a quasi-coherent sheaf on $X$. We want to consider the following two subfunctors:

$$
\Omega_{Y \rightarrow X}^{F} \subseteq \omega_{Y \rightarrow X}^{F} \subseteq{\underline{\mathrm{Quot}^{n}}}_{F_{Y} / S}^{n} .
$$




\section{WeIL RESTRICTION AND THE QUOT SCHEME}

We define $\omega_{Y \rightarrow X}^{F}$ as the subfunctor of $\underline{\text { Quot }}_{F_{Y} / S}^{n}$ whose $T$-valued points are surjective $\mathscr{O}_{Y_{T}}$-module maps $q: F_{Y_{T}} \longrightarrow \mathscr{E}$, where $\mathscr{E}$ is finite, flat of rank $n$ over $T$, such that the induced map of $\mathscr{O}_{X_{T}}{ }^{-}$ modules

$$
f_{*}(q): F_{X_{T}} \longrightarrow f_{T * \mathscr{E}}
$$

is surjective, where $f_{*}$ is the push-forward map (7.3). And we define $\Omega_{Y \rightarrow X}^{F}$ with the further requirement that the induced map of supports (7.1) be a closed immersion.

Lemma 7.9. Let $f: Y \longrightarrow X$ be a map of $S$-spaces, with $X$ separated. For any quasi-coherent sheaf $F_{X}$ on $X$ we have that $\omega_{Y \rightarrow X}^{F}$ is an open subfunctor of Quot $_{F_{Y} / S}^{n}$. Moreover, we have the following diagram of Cartesian squares:

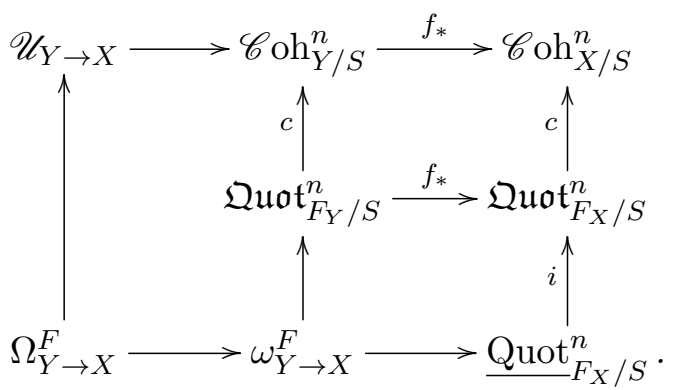

In particular, we have the following results:

(i) If $f: Y \longrightarrow X$ is an affine morphism, then the induced morphism $\mathfrak{Q u o t}_{F_{Y} / S}^{n} \longrightarrow \mathfrak{Q u o t}_{F_{X} / S}^{n}$ is schematically representable.

(ii) If $f: Y \longrightarrow X$ is affine and of finite type, then $\Omega_{Y \rightarrow X}^{F}$ is an open subfunctor of Quot $_{F_{Y} / S}^{n}$, and $\Omega_{Y \rightarrow X}^{F} \longrightarrow \underline{\text { Quot }}_{F_{X} / S}^{n}$ is schematically representable.

(iii) Finally, if $f: Y \longrightarrow X$ is affine and étale, then

$$
\Omega_{Y \rightarrow X}^{F} \longrightarrow \underline{\text { Quot }}_{F_{X} / S}^{n}
$$

is a schematically representable, étale morphism.

Proof. If $\mathscr{E}$ is a $T$-valued point of $\underline{\mathrm{Quot}}_{F_{Y} / S}^{n}$, then $f_{T *} \mathscr{E}$ has affine support over $T$ (Proposition 5.3). The surjectivity of the induced map $f_{*}(q): F_{X_{T}} \longrightarrow f_{T *} \mathscr{E}$ is then an open condition on the base, since considered as an $\mathscr{O}_{T}$-module $\mathscr{E}$ is finitely generated. This shows that $\omega_{Y \rightarrow X}^{F}$ is open in Quot $_{F_{Y} / S}^{n}$.

The three statements (i), (ii) and (iii) will all follow from Corollary 5.7 and Proposition 7.2 if we prove that the squares in the diagram are Cartesian. The upper-right square is Cartesian by Lemma 7.6. We consider the lower-right square.

We start by noting that there is a natural map $\alpha: \omega_{Y \rightarrow X}^{F} \longrightarrow P$, where $P$ is the fiber product in question. If $q: F_{Y_{T}} \longrightarrow \mathscr{E}$ is a $T$-valued point of $\omega_{Y \rightarrow X}^{F}$, then by assumption $f_{*}(q): F_{X_{T}} \longrightarrow$ $f_{T *} \mathscr{E}$ is surjective. So $\alpha(q)=\left(f_{*}(q), i(q)\right.$, id $)$. The map $\alpha$ is full and faithful.

Let $\left(q_{X}, s, \psi\right)$ be a $T$-valued point of the fiber product $P$, where $q_{X}: F_{X_{T}} \longrightarrow \mathscr{E}$ is surjective,

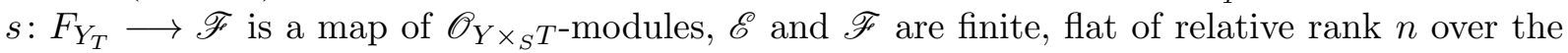




\section{R. M. SkJELnES}

base and where $\psi$ is an isomorphism of $\mathscr{O}_{X{ }_{S} T}$-modules that fits into the commutative diagram

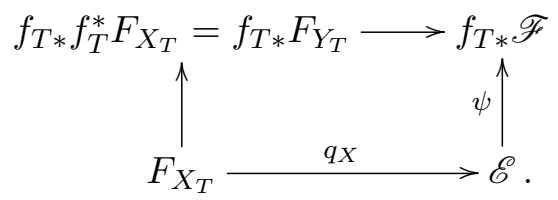

The pull-back $f_{T}^{*}\left(q_{X}\right)$ composed with the adjoint of $\psi$ gives a map

$$
q_{Y}: F_{Y_{T}}=f_{T}^{*} F_{X_{T}} \longrightarrow f_{T}^{*} \mathscr{E} \longrightarrow \mathscr{F} .
$$

We claim that $q_{Y}$ is surjective. To see this we may restrict ourselves to the support $Y^{\prime}=\operatorname{Supp}(\mathscr{F})$ of $\mathscr{F}$. By definition $Y^{\prime}$ is affine, and in fact integral over the base. Then $f: Y^{\prime} \longrightarrow X \times_{S} T$ is integral [GD61, Proposition 6.1.5], and in particular affine. Then surjectivity of $q_{Y}$ follows from surjectivity of $q_{X}$. Thus, $q_{Y}$ is a $T$-valued point of $\underline{\text { Quot }}_{F_{Y} / S}^{n}$, and in fact a $T$-valued point of $\omega_{Y \rightarrow X}^{F}$. Then $f_{*}\left(q_{Y}\right)$ is isomorphic to $\left(q_{X}, s, \psi\right)$, and thus $\alpha$ is essentially surjective.

The leftmost square is proven to be Cartesian in a similar way.

TheOREM 7.10. Let $X \longrightarrow S$ be a separated morphism of algebraic spaces, and let $F_{X}$ be a quasi-coherent sheaf on $X$. For each integer $n$, the functor $\underline{\text { unot }}_{F_{X} / S}^{n}$ is representable by a separated algebraic space.

Proof. As the Quot functor commutes with base change, we may reduce to the case where the base $S$ is an affine scheme $S=\operatorname{Spec}(A)$. Moreover, any $T$-valued point $q: F_{X_{T}} \longrightarrow \mathscr{E}$ of $\underline{\text { uuot }}_{F_{X} / S}^{n}$ is such that the $\operatorname{support} \operatorname{Supp}(\mathscr{E})$ is affine over the base. Hence the support of the quotient $\mathscr{E}$ is contained in an open quasi-compact $U \subseteq X$. Therefore, we have

$$
\lim _{\substack{U \subseteq X \\ \text { open, } q \text {-compact }}} \underline{\operatorname{Quot}}_{F_{U} / S}^{n}={\underline{\mathrm{Quot}^{n}}}_{F_{X} / S}^{n} .
$$

Hence it suffices to show the theorem for $X \longrightarrow S$ quasi-compact. With $X \longrightarrow S$ quasi-compact, we can find an affine scheme $Y \longrightarrow S$ with an étale, affine and surjective map $f: Y \longrightarrow X$. With affine schemes $Y \longrightarrow S$, the functor $\underline{\operatorname{Quot}}_{F_{Y} / S}^{n}$ is represented by a scheme [GLS07]. By Lemma 7.9(ii), the subfunctor $\Omega_{Y \rightarrow X}^{F}$ is open in $\underline{\text { uuot }}_{F_{Y} / S}^{n}$, hence a scheme. Lemma 7.9(iii) then implies that the induced map

$$
\Omega_{Y \rightarrow X}^{F} \longrightarrow{\underline{\text { Quot }^{n}}}_{F_{X} / S}^{n}
$$

is representable and étale.

We now want to see that the map (7.5) is surjective. Let $k$ be a field and let $F_{X_{k}} \longrightarrow \mathscr{E}$ be a $\operatorname{Spec}(k)$-valued point of $\underline{\operatorname{Quot}}_{F_{X} / S}^{n}$, where we use the notation $X_{k}=X \times_{S} \operatorname{Spec}(k)$. We want to show that there exists a separable field extension $k \longrightarrow L$ such that the corresponding $\operatorname{Spec}(L)$-valued point lifts to $\Omega_{Y \rightarrow X}^{F}$.

The reduced support $Z=|\operatorname{Supp}(\mathscr{E})|$ is a disjoint union of a finite set of points, given by finite field extensions $k \longrightarrow k_{i}$ with $i=1, \ldots, m$. Then $f^{-1}\left(\operatorname{Spec}\left(k_{i}\right)\right)$ is also a finite union of points $\sqcup_{j_{i}=1}^{m_{i}} \operatorname{Spec}\left(L_{j_{i}}\right)$, with $k_{i} \longrightarrow L_{j_{i}}$ a finite separable field extension for $j_{i}=1, \ldots, m_{i}$. There exists a finite separable field extension $k \longrightarrow L$ such that the induced map $k_{i} \otimes_{k} L \longrightarrow L_{j_{i}} \otimes_{k} L$ splits for all $i=1, \ldots, m$ and all $j_{i}=1, \ldots, m_{i}$. Then

$$
f^{-1}(Z) \times_{\operatorname{Spec}(k)} \operatorname{Spec}(L) \longrightarrow Z \times_{\operatorname{Spec}(k)} \operatorname{Spec}(L)
$$




\section{WeIL RESTRICTION AND THE QUOT SCHEME}

has a section, and the corresponding $\operatorname{Spec}(L)$-valued point of $\underline{\operatorname{Quot}}_{F_{X} / S}^{n}$ lifts to $\Omega_{Y \rightarrow X}^{F}$. We have then proven surjectivity, and consequently, accordingly to the definition in [RG71], proven that $\underline{\text { Quot }}_{F_{X} / S}^{n}$ is an algebraic space. That the algebraic space representing Quot ${ }_{F_{X} / S}^{n}$ is separated follows from Lemma 5.8 and the Cartesian diagram (7.4).

Remark 7.11. For $F_{X}=\mathscr{O}_{X}$, the structure sheaf on $X$, the Quot functor $\underline{\text { Quot }}_{F_{X} / S}^{n}$ is the Hilbert functor $\underline{H i l b}_{X / S}^{n}$. The situation with the Hilbert scheme was considered in [ES14], and a similar approach for Hilbert stacks was carried out in [Ryd11]. Note that when $F_{X}=\mathscr{O}_{X}$, by Proposition 6.14, we have $\omega_{Y \rightarrow X}^{F}=\Omega_{Y \rightarrow X}^{F}$; hence the situation covered in the present article generalizes the Hilbert scheme construction; see [ES14, Proposition 7.2].

Remark 7.12. The assumption that $X \longrightarrow S$ is separated is a necessary condition for representability [LS08]. On the other hand, there exist examples of separated schemes $X \longrightarrow S$ for which the Quot functor is represented not by a scheme [Knu71], but only by an algebraic space. Thus when considering representability, the setting with separated algebraic spaces $X \longrightarrow S$ is the natural one.

Remark 7.13. The above result in its generality is not covered by the result of Artin [Art69]; we have no restriction on the base space $S$, and we do not assume that $X \longrightarrow S$ is of locally finite type.

\section{ACKNOWLEDGEMEnTS}

Comments and corrections from Dan Laksov and David Rydh were important for the presentation of this manuscript. Discussions with Runar Ile about non-commutative ring theory were also helpful and clarifying.

\section{REFERENCES}

AGV73 M. Artin, A. Grothendieck and J.-L. Verdier, Séminaire de Géométrie Algébrique du Bois-Marie 1963-1964 (SGA 4), Vol. 3, Théorie des topos et cohomologie étale des schémas, Lecture Notes in Math., vol. 305, Springer-Verlag, Berlin - New York, 1973; http://dx.doi.org/10.1007/ $\mathrm{BFb} 0070714$.

Art69 M. Artin, Algebraization of formal moduli. I, Global Analysis (Papers in Honor of K. Kodaira), Univ. Tokyo Press, Tokyo, 1969, 21-71.

Art74 , Versal deformations and algebraic stacks, Invent. Math. 27 (1974), no. 3, 165-189; http://dx.doi.org/10.1007/BF01390174.

BLR90 S. Bosch, W. Lütkebohmert and M. Raynaud, Néron models, Ergeb. Math. Grenzgeb. (3), vol. 21, Springer-Verlag, Berlin, 1990; http://dx.doi.org/10.1007/978-3-642-51438-8.

Bou98 N. Bourbaki, Algebra I. Chapters 1-3, Elements of Math., Springer-Verlag, Berlin, 1998.

DB14 G. Di Brino, The quot functor of a quasi-coherent sheaf, arXiv:1212.4544v3.

Die62 J. Dieudonné, Algebraic geometry, Department of Math. Lecture Notes, No. 1, University of Maryland, College Park, Md., 1962.

ES14 T. Ekedahl and R. Skjelnes, Recovering the good component of the Hilbert scheme, Ann. of Math. (2) 179 (2014), no. 3, 805-841; http://dx.doi.org/10.4007/annals.2014.179.3.1.

GD61 A. Grothendieck and J. Dieudonné, Éléments de géométrie algébrique. II. Étude globale élémentaire de quelques classes de morphismes, Publ. Math. Inst. Hautes Études Sci. 8 (1961), 5-222; http://www.numdam.org/item?id=PMIHES_1961__8__5_0. 


\section{R. M. SkJELnES}

GD66 Éléments de géométrie algébrique. IV. Étude locale des schémas et des morphismes de schémas. troisième partie, Publ. Math. Inst. Hautes Études Sci. 28 (1966), 5-255; http: //www. numdam.org/item?id=PMIHES_1966__28__5_0.

GLS07 T.S. Gustavsen, D. Laksov and R. M. Skjelnes, An elementary, explicit, proof of the existence of Quot schemes of points, Pacific J. Math. 231 (2007), no. 2, 401-415; http://dx.doi.org/10. 2140/pjm.2007.231.401.

Gro95 A. Grothendieck, Techniques de construction et théorèmes d'existence en géométrie algébrique. IV. Les schémas de Hilbert, Séminaire Bourbaki, Vol. 6, Soc. Math. France, Paris, 1995, Exp. No. 221, 249-276.

HR15 J. Hall and D. Rydh, General Hilbert stacks and Quot schemes, Michigan Math. J. 64 (2015), no. 2, 335-347; http://dx.doi.org/10.1307/mmj/1434731927.

Knu71 D. Knutson, Algebraic spaces, Lecture Notes in Math., vol. 203, Springer-Verlag, Berlin - New York, 1971; http://dx.doi.org/10.1007/BFb0059753.

Lie06 M. Lieblich, Remarks on the stack of coherent algebras, Int. Math. Res. Not. 2006 (2006), Art. ID 75273,1-12; http://dx.doi.org/10.1155/IMRN/2006/75273.

LMB00 G. Laumon and L. Moret-Bailly, Champs algébriques, Ergeb. Math. Grenzgeb. (3) , vol. 39, Springer-Verlag, Berlin, 2000; http://dx.doi.org/10.1007/978-3-540-24899-6.

LS08 C. Lundkvist and R. Skjelnes, Non-effective deformations of Grothendieck's Hilbert functor, Math. Z. 258 (2008), no. 3, 513-519; http://dx.doi.org/10.1007/s00209-007-0183-7.

Ols06 M. C. Olsson, Hom-stacks and restriction of scalars, Duke Math. J. 134 (2006), no. 1, 139-164; http://dx.doi.org/10.1215/S0012-7094-06-13414-2.

RG71 M. Raynaud and L. Gruson, Critères de platitude et de projectivité. Techniques de "platification" d'un module, Invent. Math. 13 (1971), no. 1-2, 1-89; http://dx.doi.org/10.1007/BF01390094.

Ryd08 D. Rydh, Families of cycles and the Chow scheme, Ph.D. Thesis, KTH Royal Institute of Technology, Stockholm, Sweden, 2008, available at https://people.kth.se/ dary/thesis/thesis. pdf.

Ryd11 , Representability of Hilbert schemes and Hilbert stacks of points, Comm. Algebra 39 (2011), no. 7, 2632-2646; http://dx.doi.org/10.1080/00927872.2010.488678.

Ryd15_, Noetherian approximation of algebraic spaces and stacks, J. Algebra 422 (2015), 105147; http://dx.doi.org/10.1016/j.jalgebra.2014.09.012.

Roy Mikael Skjelnes skjelnes@kth.se

Department of Mathematics, KTH, 10044 Stockholm, Sweden 